\title{
Quantum phase via the angular momentum
}

\author{
Alexander S. Shumovsky ${ }^{1}$ \\ Department of Physics, Bilkent University, 06533 Ankara. Turkey
}

Received 5 September 1996; accepted 6 November 1996

\begin{abstract}
An approach to the quantum phase, taking into account the process of light generation and extending in this way the operational approach, is proposed. The cosine and sine operators of the phase difference of the two circularly polarized modes are determined with the aid of a polar decomposition of the angular momentum of radiative transition and conservation of the total angular momentum. Application of the approach to the Jaynes-Cummings model for an electric dipole transition shows consistency with the classical definition of the phase difference and with the results of standard operational approach as well.
\end{abstract}

PACS: $42.50 .-\mathrm{p} ; 42.50 . \mathrm{Dv}$

Keywords: Quantum phase; Operational approach; Jaynes-Cummings model; Stokes operators

The main idea of the operational approach to the quantum phase of light $[1-3]$ is to define the phase operator in terms of quantities that can be measured in real experiments. It should be stressed that the polarization measurements represent an important method of operational definition of the phase difference between two circularly orthogonal polarized modes [2-5]. The analysis of various schemes of measurement has shown that there is no unique phase operator at all [2]. Then, consideration of the so-called intrinsic and operational observables [6] has lead to the conclusion that there should be a unique intrinsic phase operator corresponding to the set of operational quantities (see also discussion in Refs. [7-9]). Further analysis of the relation between the operational approach and the method, based on the phase distributions $[10,11]$ has shown that the moments of periodic functions of the measured phase difference are those calculated from the appropriate integrated Wigner or $Q$ functions [12-14].

All these results are based on the consideration of a

\footnotetext{
'E-mail: shumo@fen.bilkent.edu.tr. Also at: Bogolubov Laboratory of Theoretical Physics, Joint Institute for Nuclear Research, Dubna, Russia.
}

photon counting measurement which includes interaction of light with a macroscopic detecting device. In this case, it is supposed that the light is prepared initially in some quantum state. Undoubtedly, this state is a result of some interactions. The process of generation of photons by a microscopic source such as an atom is the most important among them. Therefore, it seems to be quite natural to extend the operational approach and examine the phase problem in terms of what can be generated.

The aim of this paper is to discuss the relation between the phase properties of an atomic transition responsible for the generation of photons and the phase difference of the two circularly orthogonal polarizations. The phase properties of the atomic radiative transition can be easily defined by a polar decomposition of corresponding SU(2) algebra [15], describing the angular momentum of the transition. Any photon in the process of generation takes away the angular momentum of the radiative transition responsible for the creation of this photon, so that the total angular momentum is conserved [16]. Unfortunately, the polar decomposition of $\mathrm{SU}(2)$ algebra cannot be performed in the case of photons. The point is that there is no isotype [17] representation of a sub-algebra SU(2) in the WeylHeisenberg algebra describing the photons. In other words, the Casimir operator of an enveloping algebra cannot be 
uniquely determined in the whole Hilbert space corresponding to the photons.

Let us stress here, that although the recent attempt $[5,18]$ to use the polar decomposition of the Stokes operators first in an arbitrary subspace $\mathscr{H}^{(n)}$ and then to extend the definition of phase operators on the whole Hilbert space by summation over index $n$, describing the total number of photons, from $n=0$ to $x$ have led to some interesting physical results, it cannot be considered as a rigorous mathematical construction because the uniqueness of this decomposition is not proven.

At the same time, the conservation of the total angular momentum can be used in order to define the quantum phase difference of light in the operational meaning. A simple example of some considerable interest, illustrating the above statement, is provided by an atom interacting with the cavity field. This system is described by the Jaynes-Cummings model, a convenient form of which is afforded by taking the basis of spherical photons [19].

Suppose that a dipole transition is responsible for the creation of the cavity photons. Then, the angular momentum of the transition can be represented as follows

$$
\begin{gathered}
J_{i}=R_{++}-R_{--}, \quad J_{+}=\sqrt{2}\left(R_{+0}+R_{0-}\right), \\
J_{-}=\sqrt{2}\left(R_{0+}+R_{-0}\right),
\end{gathered}
$$

where $\left.R_{m m^{\prime}}=\| j=1 ; m\right\rangle\left\langle j=1 ; m^{\prime} \|,(m=0, \pm 1)\right.$ are the atomic operators. It is not difficult to see that the operators (1) obey the standard commutation relations for $\mathrm{SU}(2)$ algebra. By performing a similar analysis to that described in the Ref. [15], we can construct the polar decomposition of (1) and determine the cosine and sine operators of the Hermitian atomic phase in the form

$$
\begin{aligned}
& \hat{C}=\frac{1}{2 \sqrt{2}}\left(J_{+}+J_{-}\right)+\frac{1}{2}\left(\mathrm{e}^{i \psi} R_{-+}+\mathrm{e}^{-i \psi} R_{+-}\right), \\
& \hat{S}=\frac{1}{2 i \sqrt{2}}\left(J_{+}-J_{-}\right)+\frac{1}{2 i}\left(\mathrm{e}^{i \psi} R_{-+}-\mathrm{e}^{-i \psi} R_{+-}\right),
\end{aligned}
$$

where $\psi$ is an arbitrary real parameter (the reference phase). It is clear that $\hat{C}^{2}+\hat{S}^{2}=1$ and $[\hat{C}, \hat{S}]=0$, and the constructions (2) are Hermitian operators.

In turn, the angular momentum of the cavity field is represented by the following generators

$$
\begin{gathered}
M_{z}=\sum_{m=-1}^{+1} m a_{m}^{+} a_{m}, \quad M_{+}=\sqrt{2}\left(a_{+}^{+} a_{0}+a_{0}^{+} a_{-}\right), \\
M_{-}=\sqrt{2}\left(a_{0}^{+} a_{+}+a_{-}^{+} a_{0}\right),
\end{gathered}
$$

where $a_{m}^{+}, a_{m}$ are the operators of spherical photons with angular momentum 1 and projection $m$. Since the total angular momentum of the system described by the standard Hamiltonian

$$
H=\frac{1}{2 \mu}\left(p-\frac{e}{c} A\right)^{2}+U+\frac{1}{8 \pi} \int \mathrm{d} r\left(E^{2}+B^{2}\right)
$$

is conserved [16], one can expect that the operators chosen to represent cosine and sine of the phase of the angular momentum of the dipole radiation are of the form

$\hat{\mathscr{E}}=\frac{K_{c}}{2}\left(\frac{M_{+}+M_{-}}{\sqrt{2}}+\mathrm{e}^{i \psi} a_{-}^{+} a_{+}+\mathrm{e}^{-i \psi} a_{+}^{+} a_{-}\right)$,

$\hat{\mathscr{S}}=\frac{K_{s}}{2 i}\left(\frac{M_{+}-M_{-}}{\sqrt{2}}+\mathrm{e}^{i \psi} a_{-}^{+} a_{+}-\mathrm{e}^{-i \psi} a_{+}^{+} a_{-}\right)$,

such that $\hat{C}+\hat{\mathscr{C}}=$ const, $\hat{S}+\hat{\mathscr{S}}=$ const. Here $K_{c}, K_{s}$ are constants. One can see that $[\hat{\mathscr{C}}, \hat{\mathscr{S}}]=0$ so that the cosine and sine (4) can be measured at once.

Let us stress here some likeness between the operators (4) and the operational cosine and sine which have been determined in Ref. [2] for the measurement scheme 1 and which we denote here as $\hat{C}_{1}$ and $\hat{S}_{1}$ respectively. In fact, the operators (4) can be formally represented as

$\hat{\mathscr{C}}=\frac{K_{c}}{2}\left(\hat{C}_{1}+\frac{M_{+}+M_{-}}{\sqrt{2}}\right)$,

$\hat{\mathscr{S}}=\frac{K_{s}}{2}\left(\hat{S}_{1}+\frac{M_{+}-M_{-}}{\sqrt{2}}\right)$,

if we choose $\psi=\Delta+\pi / 2$ where $\Delta$ is the classical phase difference considered in Ref. [2] for the scheme 1. Then, it can be seen that the additional terms $\left(M_{+} \pm M_{-}\right) / \sqrt{2}$ correct, in some sense, the commutation relations for $\hat{C}_{1}$, $\hat{S}_{1}$ which are originally similar to the Susskind-Glogower relations [20] (see also Ref. [21]). It should be also mentioned that the numerical coefficients $K_{c}, K_{s}$ are determined here in a standard way [2,21] to keep the expectation values of the operators (4) lying between -1 and +1 and to have the unit value for the sum of squared averages.

In order to illustrate the above approach and clarify the physical meaning of the operators (4), let us specify the transition under consideration as an electric dipole transition and suppose that the cavity photons propagate along the $Z$-axis. Then, due to the selection rules the transition $(j=1 ; m=0) \leftrightarrow\left(j^{\prime}=0 ; m^{\prime}=0\right)$ is forbidden [19]. Under this condition, an effective Hamiltonian of the Jaynes-

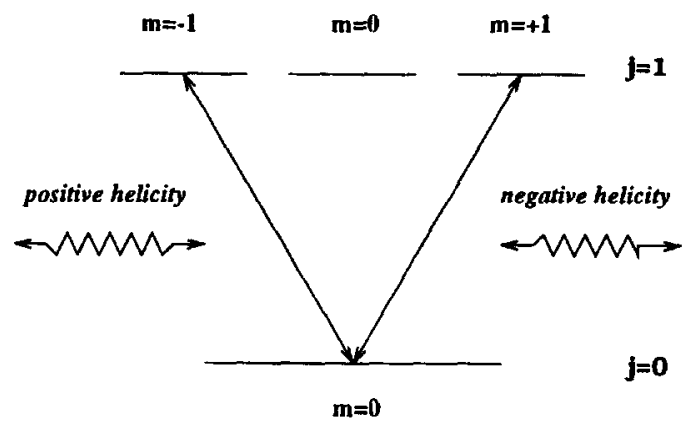

Fig. 1. Two-level atom with the electric dipole transition interacting with two circularly polarized modes of the cavity field. 
Cummings model is equivalent to a degenerated $V$-type two-level and two-mode system [22] (Fig. 1). With the assumption that the initial state of the system is a coherent mixture of two excited sub-levels of the atom and a vacuum state of the cavity field, the time-dependent wave function can be chosen as

$$
\begin{aligned}
|\Psi(t)\rangle= & \sum_{l=1}^{2} \Lambda_{l} \mathrm{e}^{-i E_{l} l} \\
& \left.\times\left[A_{l}(p \| 1 ;+1\rangle+q \| 1 ;-1\right\rangle\right)|0,0,0\rangle \\
& \left.\left.+B_{l}|| 0 ; 0\right\rangle(p|1,0,0\rangle+q|0,0,1\rangle)\right],
\end{aligned}
$$

where $p, q$ are complex numbers such that $|p|^{2}+|q|^{2}=1$. Here $\| 1, \pm 1\rangle$ denotes the atomic state with the angular momentum 1 and projections \pm 1 while $\left|n_{+}, n_{0}, n_{-}\right\rangle$ $\equiv \prod_{m}\left|n_{m}\right\rangle$ is the product of the number states for the cavity photons with given projection of the angular momentum. Assuming for simplicity exact resonance, we obtain $E_{l}=\omega \pm g, A_{l}=A_{l}= \pm 1 / \sqrt{2}, B_{l}=1 / \sqrt{2}$ where $\omega$ is the transition frequency and $g$ is the coupling constant. Then, the vacuum-field induced Rabi oscillations of cosine and sine (4) are described as follows

$$
\begin{aligned}
\langle\hat{\mathscr{C}}\rangle_{t} & =\frac{1}{2}\left\langle\left(\mathrm{e}^{i \psi} a_{-}^{+} a_{+}+\mathrm{e}^{-i \psi} a_{+}^{+} a_{-}\right)\right\rangle_{t} \\
& =\frac{|p q|}{2}(1-\cos 2 g t) \cos (\delta+\psi), \\
\left\langle\hat{\hat{J}^{\prime}}\right\rangle_{1} & =\frac{1}{2 i}\left\langle\left(\mathrm{e}^{i \phi} a_{-}^{+} a_{+}-\mathrm{e}^{-i \psi} a_{+}^{+} a_{-}\right)\right\rangle_{1} \\
& =\frac{|p q|}{2}(1-\cos 2 g t) \sin (\delta+\psi),
\end{aligned}
$$

where $\delta \equiv \arg p-\arg q$.

Thus, the evolution of the cosine and sine (4) is completely determined by the parameters of the atomic system. Taking into account that the operators $a_{+}, a_{-}$correspond to the photons with negative and positive helicity respectively, it is not difficult to see that the expectation values (5) coincide with the Stokes parameters $s_{1}$ and $s_{2}$ determined as the averages of the Stokes operators in the circularly polarized basis [23]. Therefore. the expressions (5) determine the classical cosine and sine of the phase difference between two circularly polarized modes of the cavity field in terms of the atomic parameter $\delta$. We now note that due to the specification of the system, the $\left(M_{+} \pm\right.$ $\left.M_{-}\right) / \sqrt{2}$ terms do not contribute to the expectation values (5) so that these expectation values formally coincide with the averages of the operational cosine and sine $\hat{C}_{1}$ and $\hat{S}_{1}$ [2]. However, unlike the case of the operational scheme 1, both the cosine and sine (4) can be measured at once.

In conclusion, let us briefly summarize the results. The description of the quantum phase properties of light in terms of the polar decomposition of the angular momentum using the conservation of the total angular momentum in the system "atom + radiation", is proposed. Within the framework of this approach, the cosine and sine of the phase difference of the two circularly orthogonal polarizations are determined in terms of the angular momentum of the field. Investigation of the electric dipole transition shows that the above approach is consistent both with the classical definition of the phase difference in terms of the Stokes parameters and with the operational definition of the phase in the standard form. This consideration can be extended to an atom with transitions of different type and to multi-atom systems. Let us emphasize that in the spirit of the philosophy of Ref. [6], one can expect that the quantum phase of the angular momentum may play the role of an intrinsic observable corresponding to the measured operational phase. Detailed investigation of this aspect as well as the relation among the above approach, the operational approach, and the method derives the phase distribution via appropriate phase-space functions $[10,14]$ needs further discussion.

The author wishes to thank Dr. A. Miranowicz for helpful discussions.

\section{References}

[1] J.W. Noh, A. Fougères and L. Mandel. Phys. Rev. Letl. 67 (1991) 1426.

[2] J.W. Noh, A. Fougères and L. Mandel, Phys. Rev. A 45 (1992) 424.

[3] J.W. Noh, A. Fougères and L. Mandel, Phys. Scripta T 48 (1993) 29

[4] T. Hakioğlu, A.S. Shumovsky and O. Aytür, Phys. Lett. A 194 (1994) 304

[5] A. Luis, L.L. Sànchez-Soto and R. Tanaș, Phys. Rev. A 51 (1995) 1634.

[6] B.-G. Englent and K. Wódkiewicz, Phys. Rev. A 51 (1995) R266.

[7] Special issue on: Quantum Phase and Phase Dependent Measurements, Phys. Scripta T 48 (1993).

[8] R. Lynch, Phys. Rep. 256 (1995) 367.

[9] R. Tanaś, A. Miranowicz and Ts. Gantsog. in: Progress in Optics, Vol. 35, ed, E. Wolf (North-Holland, Amsterdam. 1996).

[10] A. Bandilla and H. Paul, Ann. Phys. (Leipzig) 23 (1969) 323.

[11] W. Schleich, R.J. Horwicz and S. Varro, in: Quantum Optics V, eds. D.F. Walls and J. Harvey (Springer, Heidelberg, 1989).

[12] M. Freyberger and W. Schleich, Phys. Rev. A 47 (1993) R.30.

[13] U. Leonhardt and H. Paul, Phys. Rev. A 47 (1993) R2460.

[14] M. Freyberger, K. Vogel and W. Schleich, Phys. Lett. A 176 (1993) 41

[15] A. Vourdas, Phys. Rev. A 41 (1990) 1653.

[16] C. Cohen-Tannoudji, J. Dupont-Roc and G. Grynberg. Photons and Atoms (Wiley, New York, 1989).

[17] J.-P. Serre, Linear Representations of Finite Groups (Springer, New York, 1977). 
[18] A. Luis and L.L. Sánchez-Soto, Phys. Rev. A 48 (1993) 4702.

[19] W. Heitler, The Quantum Theory of Radiation (Dover, New York, 1984).

[20] L. Susskind and J. Glogower, Physics 1 (1964) 49.

[21] P. Carruthers and M.M. Nieto, Rev. Mod. Phys. 40.

[22] See, e.g., the review articles: H.I. Yoo and J.H. Eberly, Phys.
Rep. 118 (1985) 239; F.L. Kien and A.S. Shumovsky, Int. I. Mod. Phys. B 5 (1991) 2287; R. Shore and P.L. Knight, J. Mod. Optics 40 (1993) 1195.

[23] Our notations of the Stokes parameters are that of $M$. Born and E. Wolf, Principles of Optics (Pergamon Press. Oxford, 1984). 